\title{
Guidance for Head and Neck clinical trials during COVID-19
}

\author{
Pankaj Chaturvedi, MS, FACS ${ }^{1}$
}

${ }^{1}$ Tata Memorial Center

April 28, 2020

\begin{abstract}
As this ever-evolving pandemic lays itself, more of its impact is being understood. Until recently, most guidelines were reported to aid in managing and treating suspected or confirmed cases. Research institutions around the world are responding with a sense of confusion. Some are continuing routinely, especially those who are overseeing clinical trials that could offer life-saving therapies, particularly against the novel coronavirus. Since research must continue even in the face of a shutdown, we aim to collate the currently available recommendations from various organizations and provide guidance to head and neck researchers across the world during these trying times.
\end{abstract}

\section{Authors:}

Arjun Gurmeet Singh, MDS

(Oral and Maxillofacial Surgery), MFDS (Glasgow)

Department of Head and Neck Oncology

Tata Memorial Center

Mumbai, India

Email: arjun193@gmail.com

Pankaj Chaturvedi, MS, FACS (Corresponding author)

Department of Head and Neck Oncology

Tata Memorial Center

Mumbai, India

Email: chaturvedi.pankaj@gmail.com

Funding statement

No funding was obtained from any sources.

\section{Conflict of Interest statement}

None of the authors have any conflict of interest to declare.

\section{Authors contribution statement}

All authors have made a significant contribution to this article from concept to implementation and publication.

\section{Abstract}


As this ever-evolving pandemic lays itself, more of its impact is being understood. Until recently, most guidelines were reported to aid in managing and treating suspected or confirmed cases. Research institutions around the world are responding with a sense of confusion. Some are continuing routinely, especially those who are overseeing clinical trials that could offer life-saving therapies, particularly against the novel coronavirus. Since research must continue even in the face of a shutdown, we aim to collate the currently available recommendations from various organizations and provide guidance to head and neck researchers across the world during these trying times.

Introduction

Various challenges exist for researchers during the Coronavirus pandemic that are resulting from restrictions at healthcare facilities, changes in availability of staff and unidentified possible interactions. Some trial participants or investigators might also be required to self-isolate or be infected by the severe acute respiratory syndrome-like coronavirus-2 (SARS-CoV-2) causing a moral dilemma in keeping trials running. Another issue specific to the head and neck region is the proximity to the areas through which the virus can be easily transmitted requiring further amendments to protocols and need for additional equipment. Multi institutional trial conduct can also be affected by the unavailability of Investigational Medicinal Products (IMPs) due to the restrictions affecting supply chains across the world. All these factors might have an impact on the accrual, assessment and provision of clinical trials.

The impact of Coronavirus pandemic needs to be considered on ongoing and new clinical trials. Sponsors need to take into account the national guidelines and restrictive measures imposed including limitations of trial participants and staff confinements and their ability to perform visits, interviews and forms and notification of adverse effects. Funding agencies and sponsors should be informed regarding the impact the current situation might have on the trial protocol. Currently, most of the trials are being run based on risk-benefit concerns and contingencies present locally with the highest priority given to the health and safety of the trial participants. In the event the participant is unable to attend a scheduled visit, measures such as virtual or tele contact may be required to identify ensure adequate medical oversight. With all these limitations it is of utmost importance to make provisions for data protection with adequate documentation. It is also important to note that international publishers have made clear that in the event of a public health emergency, information with immediate health implications would be disseminated without any restrictions. This document aims to collate the currently available recommendations from various organizations and provide guidance to head and neck researchers across the world during these trying times.

Initiating new trials

Regulatory bodies have currently paused approvals for non-COVID-19 new trial registration unless requiring an expedited approval in the event of public health emergencies with few also advising to withhold enrollment.(1) The study team should critically assess the feasibility of starting a clinical trial or enrolling new participants. The risk benefit section of protocols should include the additional risks that the participant and trial workers could encounter due to COVID-19 with adequate risk mitigation measures. Most guidelines suggest a risk assessment by the sponsor weighed against the anticipated benefit for the participant and society before initiating enrollment. $(2,3)$ In trials especially of the head and neck cancer, where the participants enrolled may be determined as being a risk group for COVID-19 or in trial that involve increasing the risk to contract COVID-19 such as chemotherapy related trials, should be carefully considered before starting or continuing such trials.

Considerations for ongoing trials

Ensuring safety of trial participants is paramount and decisions should be taken on an individual participant basis focusing on the potential benefit. Decisions regarding further trial recruitment, continuing use of IMPs for enrolled participants and need to change the monitoring methods and site during an ongoing trial should be kept in mind. At any stage, it is very important for participants to be kept informed of changes to study and other plans that could impact their care. 


\section{How to obtain informed consent from patient in isolation?}

All guidelines suggest obtaining written informed consent before enrolling patients. If this is not possible due to COVID-19 isolation measures or safety, electronic methods should be considered.(4) If not possible electronically, a three way call phone or video communication with the investigator, patient and an impartial witness and/or if requested, additional participants (e.g. next in kin, spouse, etc.) have been suggested for verbal confirmation. If the signed consent form cannot be collected from the patient, an attested copy by the witness and investigator who participated in the call or a photograph of the signed informed consent with attestation of the investigator should suffice.(4) The details of the above procedure should be included with the informed consent in the source notes for records.

\section{Protection of participant and the investigators}

Clinical investigators and sponsors should consult their Institutional Review Board (IRBs) or Institutional Ethics Committees (IECs) in deciding if the participants' safety and rights are best served and protected by participating in the ongoing study or by discontinuing the IMP, intervention or even participation in the trial.

Most head and neck oncology trials are ongoing in an institutional setup where preemptive measures to contain the virus are already instituted. If the study is enrolling participants, provisions for investigator's safety should be available at the site. If not possible, the sponsor should choose to withhold further intervention or monitoring until such safety measures are in place for the investigator.

How to report changes to the ethics committee?

Most guidelines encourage sponsors and investigators to engage with their local IRB/IEC as early as possible as emergent changes are anticipated in protocols due to COVID-19.(1,2,4) Some sponsors may choose to redesign their studies to require less labor, at the same time in a way that salvages the study. The changes made to minimize the hazard or protect the life and well being of participants and investigators may be implemented without IRB approval before filing an amendment, but are required to be reported later. The amendment needs to include the specific protocol deviation and the reason for the deviation. For example, specific visits conducted by virtual contact rather than as specified in the protocol due to the current situation, generally would be acceptable. For efficacy assessments, protocol modifications are required for the collection of endpoints done via methods alternate to the ones specified in the protocol such as virtual assessments, delays and alternate collection of specimens.(4)

Are alternate forms of visit appropriate?

Since trial participants may not be able to visit the site for the protocol specific visits and investigations, sponsors should evaluate if alternate measures such as virtual visits, alternate locations for assessment, including imaging centers and labs, could suffice when necessary, only after ensuring the safety of the participant. If an in-person visit is necessary, it is the sponsors' responsibility to assure the implementation of the monitoring safely. $(2,4)$ This is important for trials that include participants who no longer have access to IMPs and who need additional safety monitoring (eg. for withdrawal of an active IMP).

\section{Should COVID-19 screening be reported?}

Some institutions might mandate screening all participants for COVID-19 as a protocol. This does not need to be reported or amended in the protocol unless the testing data would be collected and used as a part of a new objective in the study.(4)

\section{Home delivery of IMPs}

Since the scheduled visits at the site may be significantly impacted, certain IMPs that require self-administration may be amenable for home delivery but should be documented. This may include infusions as well. Where self-administration is not possible and monitoring is required, alternate administration methods via home 
nurses or at smaller care facilities may be planned after obtaining approval from the local reviewing authorities. In all cases it is important to maintain IMPs accountability before implementing any alternate methods.

Dealing with possible missing data and statistical analyses

Due to the changes in scheduled visits or patient's discontinuation, there may be missing information for the protocol specified procedures. Nonetheless, it is important to capture all details in the case report form that explains the basis of this missing information. This information should be summarized in the study report. If changes in protocol, including missing data, will lead to amending the data collection and statistical analysis, the sponsor should consider informing the local review board before locking their databases. A pre-specified plan for analyses should be constituted to tackle the protocol deviations related to COVID-19.

The need to apply new processes or to modify existing ones will vary based on the protocol and local situation. Some considerations need to be made whether it is appropriate to delay assessments for an ongoing trial or to discontinue or withdraw participants if the study cannot be properly conducted under the existing protocols.

It is important to keep the measures going that yield the most information for the least effort and resources. Sponsors should describe the measures instituted in view of the ongoing pandemic that may include:

- Contingency measures to manage study conduct during disruption

- Document and list all participants affected by COVID-19 and describe how the participation as altered

- Analyze and discuss the impact of the contingency measures on safety and efficacy

\section{References}

1. Q\&A on the impact of COVID-19 on research funded or supported by NIHR [Internet]. [cited 2020 Apr 16]. Available from: https://www.nihr.ac.uk/documents/qanda-on-the-impact-of-covid-19-on-researchfunded-or-supported-by-nihr/24467

2. guidanceclinicaltrials_covid19_en.pdf [Internet]. [cited 2020 Apr 16]. Available from: https://ec.europa.eu/health/sites/hea 10/guidanceclinicaltrials_covid19_en.pdf

3. Research C for DE and. Clinical Trial Conduct During the COVID-19 Pandemic. FDA [Internet]. 2020 Apr 7 [cited 2020 Apr 16]; Available from: https://www.fda.gov/drugs/coronavirus-covid-19-drugs/clinicaltrial-conduct-during-covid-19-pandemic

4. FDA Guidance on Conduct of Clinical Trials of Medical Products during COVID-19 Pandemic: Guidance for Industry, Investigators, and Institutional Review Boards. :16. 